\title{
Therapeutic Human Antithrombin-III
}

National Cancer Institute

\section{Source}

National Cancer Institute. Therapeutic Human Antithrombin-III. NCI Thesaurus. Code C136824.

A form of the human glycoprotein antithrombin-III (AT-III), which is produced recombinantly or isolated from human plasma, with anticoagulant activity. Upon administration, antithrombin-III binds to and blocks thrombin activity, and prevents thrombus formation. 